\title{
Author Correction: Lattice-strained metal-organic-framework arrays for bifunctional oxygen electrocatalysis
}

\author{
Weiren Cheng, Xu Zhao, Hui Su, Fumin Tang, Wei Che, Hui Zhang and Qinghua Liu(i)
}

Correction to: Nature Energy https://doi.org/10.1038/s41560-018-0308-8, published online 14 January 2019.

In the version of this Article originally published, in Fig. 1f, the pink and blue XRD curves were incorrectly labelled as ' $2.7 \%$-MOF' and ' $1.0 \%$-MOF', respectively; instead they should have been ' $3.6 \%$-MOF' and ' $1.7 \%$-MOF', respectively. Additionally, there were errors in the XRD data. The data were presented between $2 \theta$ values of $5-42^{\circ}$ and the dataset for each sample was presented as one measurement, but was actually made up of data from two sets of measurements using two $2 \theta$ ranges of $5-10^{\circ}$ and $10-42^{\circ}$ with different time collection periods of $0.5 \mathrm{~s}$ and $0.2 \mathrm{~s}$ for each single point, respectively. In assembling Fig. 1f, the high-angle data $\left(10-42^{\circ}\right)$ for the $4.3 \%$-MOF sample were mistakenly imported for both the 4.3\%-MOF and 3.6\%-MOF samples, after which smoothing operations were performed on the data. This resulted in the plots for the $4.3 \%$-MOF and 3.6\%-MOF mistakenly having the same raw data between $10-42^{\circ}$. Although the raw data were identical between $10-42^{\circ}$, there were small variations between the two plots shown for the $4.3 \%$-MOF and 3.6\%-MOF due to slight differences in the smoothing operations. The correct data for the 3.6\%-MOF sample are now included and data for each of the four samples are presented in separate graphs, in Fig. $1 \mathrm{e}$ for the $5-10^{\circ}$ range and in Fig. $1 \mathrm{f}$ for the $10-42^{\circ}$ range, to reflect the fact that two separate measurements were performed. The corrected data presented are unprocessed. The data shown in Fig. 1e were presented correctly as originally published but are now shown as unprocessed data for consistency with Fig. 1f. The original and corrected Fig. 1e,f are shown below. Accordingly, Fig. 1e,f caption has been amended: "e,f, Enlarged XRD (e) and XRD (f) patterns for the pristine, $1.7 \%-, 3.6 \%$ - and $4.3 \%$-MOFs, and the XRD simulation of the NiFe MOF (turquoise bars)." has been changed to "e,f, XRD patterns for the pristine, $1.7 \%-, 3.6 \%$ - and $4.3 \%$-MOFs in $2 \theta$ ranges of $5-10^{\circ}(\mathbf{e})$ and $10-42^{\circ}(\mathbf{f})$, and the XRD simulation of the NiFe MOF (turquoise bars)."
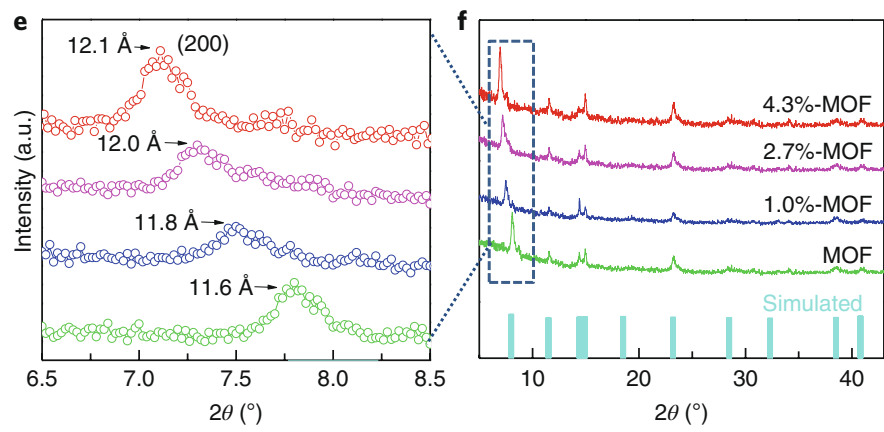

Corrected
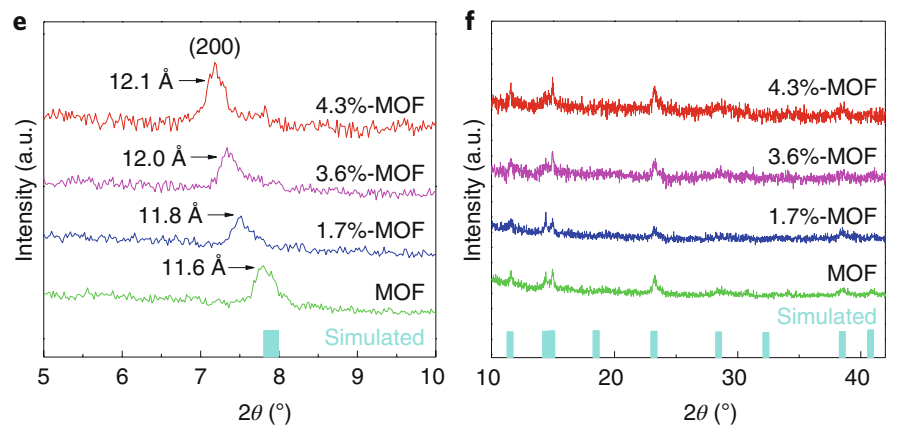

Fig. 1 | Original and Corrected. 
Furthermore, in Fig. 4c,d, the $x$-axis labels 'Binding energy $(\mathrm{eV})$ ' should have been 'Energy (eV)'. In the Methods, in the section 'Synthesis of pristine and lattice-strained MOFs', in the sentence beginning "In a typical process..., "deionized of size" should have read "Ni foam with a size of"; the correct sentence is "In a typical process, $8 \mathrm{mg}$ of $\mathrm{Ni}(\mathrm{Ac})_{2} \cdot 4 \mathrm{H}_{2} \mathrm{O}$ and $2 \mathrm{mg}$ of $\mathrm{Fe}\left(\mathrm{NO}_{3}\right) \cdot 9 \mathrm{H}_{2} \mathrm{O}$ were dissolved into $1 \mathrm{ml}$ of deionized water in a vial, and then a piece of Ni foam with a size of $2 \mathrm{~cm} \times 1 \mathrm{~cm} \times 0.5 \mathrm{~mm}$ was immersed into the above solution". And in the section 'Operando SR-FTIR spectroscopy and XAS measurements', to improve clarity of the descriptions of the XAS measurements the following sentence has been added: "To collect the $\mathrm{Ni}$ and $\mathrm{Fe}_{3,2}$-edge XANES spectra at different applied potentials, the NiFe MOF samples after the electrochemical reactions at various bias potentials of the ORR and OER were transferred into the measurement chamber of the BL12B-a beamline from the electrochemical cells".

These errors have been corrected in all versions of the Article.

Published online: 19 March 2020

https://doi.org/10.1038/s41560-020-0599-4

(c) The Author(s), under exclusive licence to Springer Nature Limited 2020 